\title{
Naloxone antagonism of hyperactivity in morphine-treated hamsters
}

\author{
PAUL SCHNUR, DAVID HANG, and AUDRA STINCHCOMB \\ University of Southern Colorado, Pueblo, Colorado
}

\begin{abstract}
Two experiments investigated the effects of amount of exposure to morphine on naloxone $(0.4 \mathrm{mg} / \mathrm{kg})$-induced changes in hamster running-wheel activity. Naloxone injections were given on the first, fifth, or ninth test day of morphine administration. In Experiment 1, naloxone was given $2 \mathrm{~h}$ after a $15-\mathrm{mg} / \mathrm{kg}$ dose of morphine. The results indicated that naloxone-precipitated hypoactivity increased across test days. In Experiment 2, naloxone was given 10 min after a 2.5$\mathrm{mg} / \mathrm{kg}$ dose of morphine. The results indicated that naloxone antagonized morphine-elicited hyperactivity and that naloxone's effects increased across test days. These findings are discussed in terms of several hypotheses of naloxone-induced changes in morphine-treated hamsters.
\end{abstract}

Opiate antagonists such as naloxone and naltrexone can block the behavioral effects of morphine in a variety of species (Goldberg, Morse, \& Goldberg, 1976; Thornhill, Hirst, \& Gowdey, 1978). That is, when morphine is administered with an opiate antagonist, its behavioral effects are prevented or diminished in a dose-dependent fashion (e.g., Downs \& Woods, 1976). Moreover, under some circumstances, opiate antagonists can reverse the behavioral effects of morphine, converting one behavioral pattern into another (Brady \& Holtzman, 1981; Marcais, Bonnet, \& Costentin, 1981). For example, working with locomotor activity in hamsters, we have demonstrated that naloxone can reverse both morphine-elicited hypoactivity and morphine-elicited hyperactivity. Compared with saline controls, morphine $(15 \mathrm{mg} / \mathrm{kg})$ elicits a biphasic time-effect pattern comprising hypoactivity, recovery, and hyperactivity (Schnur, 1985b; Schnur, Bravo, Trujillo, \& Rocha, 1983). However, when naloxone $(0.4 \mathrm{mg} / \mathrm{kg})$ is administered prior to morphine, the initial hypoactivity is reversed and hyperactivity occurs in its place (Schnur, 1985a; Schnur \& Raigoza, 1986a). Similarly, when naloxone is administered 1-2 $\mathrm{h}$ after the injection of morphine, recovery (Schnur \& Raigoza, 1986a) and/or hyperactivity (Schnur, 1985a; Schnur \& Raigoza, 1986b) are reversed and hypoactivity occurs instead. In these instances, then, naloxone does not merely negate the effects of morphine; rather, it elicits a significant level of opposite-directed activity.

It is possible to account for such findings by positing the existence of opponent processes underlying morphine's observable effects. For example, we have proposed a modified dual-action hypothesis (Schnur, 1985a; Schnur \& Raigoza, 1986a, 1986b), according to which morphine elicits two concurrent, mutually inhibitory, processes. One process mediates behavioral activation and hyperactivity;

\footnotetext{
This research was supported by NIH Minority Biomedical Research Support Grant RR-08197-04 to the University of Southern Colorado (Paul Schnur, principal investigator). The authors would like to thank Charlotte Archuletta for her assistance in the conduct of the experiments. Address correspondence to Paul Schnur, Department of Psychology, University of Southern Colorado, Pueblo, CO 81001-4901.
}

the other mediates behavioral inhibition and hypoactivity. At a particular point in time, one process predominates while its complement is masked. Naloxone acts by blocking the predominant process and unmasking its complement. Similarly, Solomon and Corbit's (1974) opponentprocess theory of motivation hypothesizes that the initial effects of a drug (the A process) elicit a counterreaction (the B process), which summates with the initial reaction to produce the final behavioral outcome. Within this framework, naloxone might be said to unmask the B process.

Naloxone reversal of morphine-elicited hyperactivity can also be accounted for in terms of dependence/withdrawal mechanisms. According to a dependence/withdrawal hypothesis, naloxone-induced hypoactivity is the result of naloxone-precipitated withdrawal in morphine-dependent animals. The plausibility of this hypothesis rests upon demonstrations in dogs (Jacob \& Michaud, 1974), mice (Stevens \& Klemm, 1979), and rats (Coussens, Crowder, \& Smith, 1973) that dependence and withdrawal symptoms are manifest after acute administration of low doses of morphine, comparable to those employed in our work with the hamster (e.g., Schnur, 1985a). The purpose of the present work was to investigate naloxone reversal of morphineelicited hyperactivity as a function of exposure to morphine.

\section{EXPERIMENT 1}

Previous work has indicated that naloxone, administered 1 or $2 \mathrm{~h}$ after a $15-\mathrm{mg} / \mathrm{kg}$ dose of morphine, produces doserelated hypoactivity (compared to saline controls) that lasts approximately $40 \mathrm{~min}$ (Schnur \& Raigoza, 1986a). If naloxone-precipitated hypoactivity is due to dependence/ withdrawal mechanisms, then the magnitude of this effect should increase as a function of exposure to morphine. Similarly, the opponent-process theory of motivation holds that the magnitude of the $B$ process increases with continued drug exposure. Therefore, naloxone-precipitated hypoactivity, to the extent that it represents an unmasking of the B process, should increase as a function of exposure to morphine. 


\section{Method}

Subjects. Thirty-two adult female golden Syrian hamsters with a mean weight of $115 \mathrm{~g}$ were used. The animals were obtained from Sasco, Inc. (Omaha, NE), housed individually in wire-mesh cages, maintained on a 12:12 light-dark cycle (lights on at 7 a.m.), and given free access to tap water and paper nesting material throughout the experiment. After each experimental session, the hamsters received a daily food ration (Purina Rodent Lab Chow) sufficient to maintain $90 \%$ of their ad-lib weights.

Apparatus and Materials. The apparatus consisted of 32 activity wheels (Wahmann Co., Model LC-34) housed in two rooms, each dimly illuminated by two $15-\mathrm{W}$ bulbs. Running wheels were fitted with microswitches and connected via interfaces (Lafayette Model 1180) to Apple II+ computers to record the number of wheel revolutions. An ambient noise level of $70 \mathrm{~dB}$ (re: 0.0002 dynes $/ \mathrm{cm}^{2}$, A scale) was maintained.

Morphine injections consisted of $15-\mathrm{mg} / \mathrm{kg}$ doses of morphine sulfate (Lilly), naloxone injections consisted of $0.4-\mathrm{mg} / \mathrm{kg}$ doses of naloxone hydrochloride (DuPont), and saline injections consisted of a $0.9 \%$ solution of sodium chloride. All injections were administered subcutaneously in the dorsal surface of the neck in $1-\mathrm{ml} / \mathrm{kg}$ volumes.

Procedure. The experiment was conducted on 12 consecutive days. On each of the first 3 days, the animals were given saline injections and placed in the running wheels for a 3-h baseline session. These sessions served to acclimate the animals to the running wheels and to the handling/injection procedures. Animals were then randomly assigned to one of four groups: MS/NLX $(n=8)$, MS/SAL $(n=8)$, SAL/NLX $(n=8)$, and SAL/SAL $(n=8)$. On Day 4 (Test Day 1$)$, the animals were tested during a 3-h session. Groups MS/NLX and MS/SAL received morphine and Groups SAL/NLX and SAL/SAL received saline $10 \mathrm{~min}$ before being placed in the running wheels. After $2 \mathrm{~h}$, the animals were removed from the wheels for the second round of injections. Groups MS/NLX and SAL/NLX received naloxone and Groups MS/SAL and SAL/SAL received saline injections $10 \mathrm{~min}$ before being replaced in the wheels for $1 \mathrm{~h}$. On Days 5, 6, and 7, Groups MS/NLX and MS/SAL received morphine and Groups SAL/NLX and SAL/SAL received saline $10 \mathrm{~min}$ before being placed in the running wheels for a 3-h session. On Day 8 (Test Day 2), the animals were tested as they had been on Day 4. On Days 9, 10, and 11, treatments were identical to those on Days 5, 6, and 7. On Day 12 (Test Day 3), the animals were tested as they had been on Days 4 and 8 . The number of wheel revolutions was recorded every $20 \mathrm{~min}$ for each animal.

\section{Results and Discussion}

Panels a, b, and c of Figure 1 show mean activity as a function of 20-min time blocks for all groups on Test Days 1, 2, and 3, respectively. Data are plotted separately for activity before and after the second injection. Morphine's biphasic time-effect pattern can be seen during the first $2 \mathrm{~h}$ of each test session. Compared with saline controls, morphine-treated animals were first hypoactive and subsequently hyperactive, although on Test Day 2, hyperactivity was inexplicably weak (cf. Schnur, Bravo, \& Trujillo, 1983). A $2 \times 3 \times 6$ (first injection $\times$ test days $\times$ time blocks) mixed factorial analysis of variance (ANOVA) of locomotor activity prior to the second injection indicated that the effect of test days was significant $[F(2,60)=16.86, p<.001]$, as was the effect of time blocks $[F(5,150)=50.74, p<.001]$. In addition, the interactions between the first injection and time blocks $[F(5,150)=18.48, p<.001]$, between test days and time blocks $[F(10,300)=2.91, p<.005]$, and between the first injection, test days, and time blocks $[F(10,300)$ $=2.74, p<.005]$ were significant.

The effects of naloxone on morphine-elicited activity can be seen during the last hour of each test session. Looking across panels, it is evident that naloxone-precipitated hypoactivity increased as a function of morphine adminis-
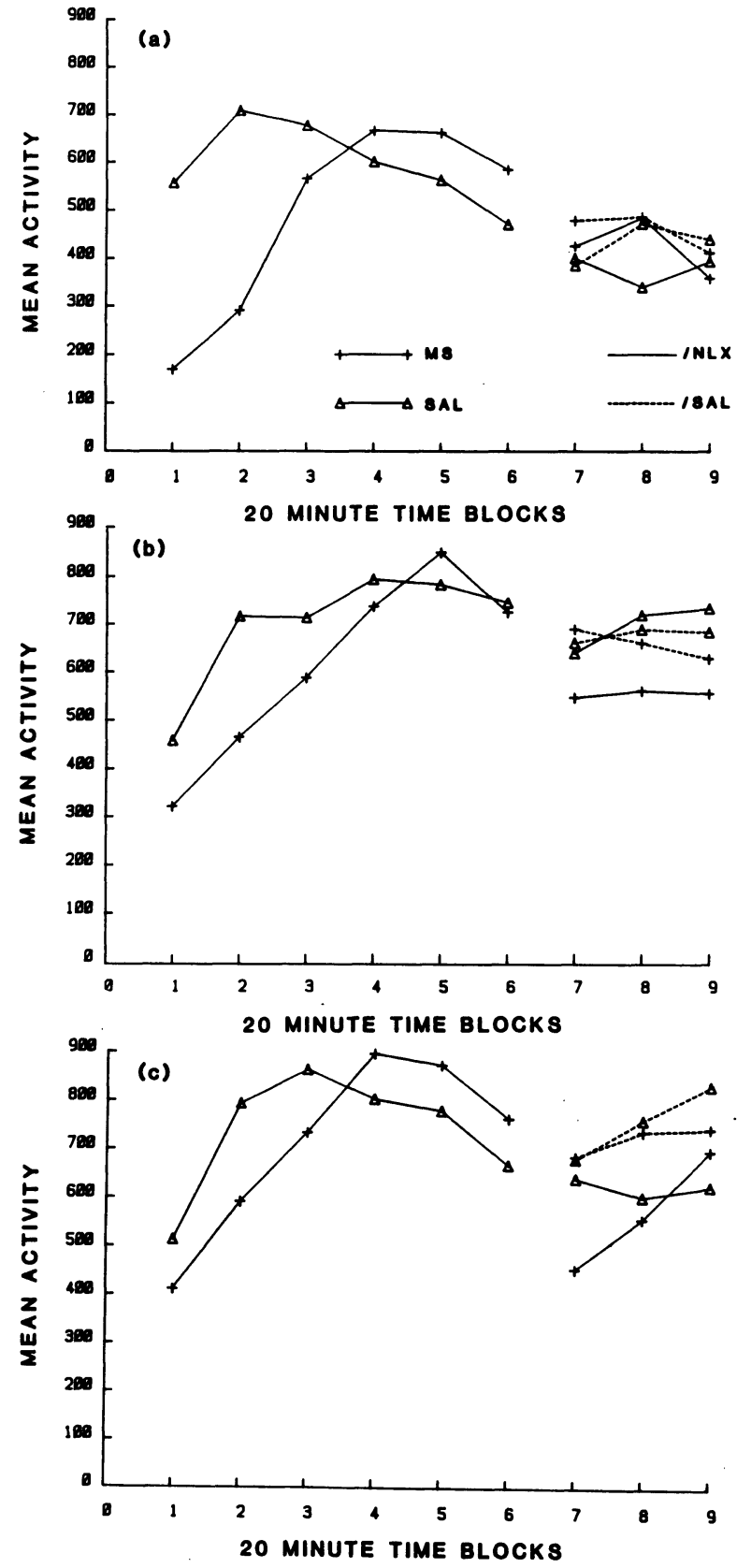

Figure 1. Mean running-whed activity as a function of $20-\mathrm{min}$ time blocks for all groups in Experiment 1. Punels a, b, and c show results on Test Days 1, 2, and 3, respectively. For each test day, data are plotted separately before and after the second injection.

tration. On Test Day 1, naloxone had no measurable effect: Locomotor activity in Group MS/NLX was not different from that in the other groups. On Test Day 2, however, naloxone produced hypoactivity: Following the naloxone injection, Group MS/NLX was hypoactive compared with all other groups. During the $20 \mathrm{~min}$ immediately following the second injection, Group MS/NLX was approximately $19 \%$ less active than the other groups. Similarly, on Test Day 3, Group MS/NLX was hypoactive: During the $20 \mathrm{~min}$ immediately following the sec- 
ond injection, Group MS/NLX was approximately $34 \%$ less active than the other groups.

A $2 \times 2 \times 3 \times 6$ (first injection $\times$ second injection $\times$ test days $\times$ time blocks) mixed factorial ANOVA of locomotor activity after the second injection indicated that the effect of test days was significant $[F(2,56)=19.52$, $p<.001]$. In addition, the interaction between test days and time blocks was significant $[F(4,112)=3.84$, $p<.01]$. Finally, the interactions between the first injection, test days, and time blocks $[F(4,112)=2.35]$ and between the first injection, the second injection, test days, and time blocks $[F(4,112)=2.35]$ were marginally significant $(p \mathrm{~s}<.06)$. Newman-Keuls tests $(p<.05)$ were conducted to test differences among groups following the second injection on Test Days 1,2, and 3. On Test Day 1, there were no differences among groups during the first $20 \mathrm{~min}$ after the second injection. On Test Day 2, however, Group MS/NLX was less active than Group MS/SAL, and on Test Day 3, Group MS/NLX was less active than all other groups, during the same $20 \mathrm{~min}$. Forty minutes after the second injection, Group SAL/NLX was less active than all other groups on Test Day 1. During the same time period, Group MS/NLX was less active than Groups SAL/NLX and SAL/SAL on Test Day 2 and Group MS/NLX was less active than Groups MS/SAL and SAL/SAL on Test Day 3. Sixty minutes after the second injection, there were no differences among groups on Test Day 1. During the same time period, however, Group MS/NLX was less active than either Group SAL/SAL or Group SAL/NLX on Test Day 2, and on Test Day 3, Groups MS/NLX and SAL/NLX were less active than Group SAL/SAL.

These results indicate that naloxone-induced hypoactivity in morphine-treated animals increases as a function of exposure to morphine. Naloxone precipitated more hypoactivity in morphine-treated animals on Test Day 3 than on Test Days 1 and 2, an outcome correctly anticipated by both the dependence/withdrawal hypothesis and the opponent-process theory of motivation outlined above. However, it should be noted that support for these hypotheses derives principally from results obtained during the 20 min immediately following the second injection. This may reflect the short half-life of naloxone's actions, but if that is so, the difference in the apparent duration of naloxone's effects on Test Days 2 and 3 cannot be reconciled easily.

\section{EXPERIMENT 2}

Experiment 2 tested the effects of naloxone on hyperactivity elicited by a low dose $(2.5 \mathrm{mg} / \mathrm{kg})$ of morphine. Experiment 2 also tested the effects of exposure to morphine on naloxone antagonism of low-dose hyperactivity. As in the first experiment, a naloxone $(0.4 \mathrm{mg} / \mathrm{kg})$ challenge was given on the first, fifth, and ninth days of morphine $(2.5 \mathrm{mg} / \mathrm{kg})$ administration. If naloxone antagonism of morphine hyperactivity is due to dependence/withdrawal mechanisms, or if it represents an unmasking of the opponent $B$ process, then the magnitude of this effect should increase as a function of exposure to morphine.

\section{Method}

Subjects. Thirty-two adult golden Syrian hamsters ( 13 males, $19 \mathrm{fe}$ males) descended from animals obtained from Sasco, Inc. (Omaha, NE), with a mean weight of $104 \mathrm{~g}$, were used. Conditions of housing and maintenance were identical to those in Experiment 1.

Apparatus and Materials. The apparatus was identical to that used in Experiment 1. Morphine injections consisted of $2.5-\mathrm{mg} / \mathrm{kg}$ doses of morphine sulfate (Lilly), naloxone injections consisted of $0.4-\mathrm{mg} / \mathrm{kg}$ doses of naloxone hydrochloride (DuPont), and saline injections consisted of a $0.9 \%$ solution of sodium chloride. All injections were administered subcutaneously in the dorsal surface of the neck in $1-\mathrm{ml} / \mathrm{kg}$ volumes.

Procedure. The experiment was conducted on 12 consecutive days. The procedure was similar to that of Experiment 1. Days 1-3 were saline baseline sessions as described in Experiment 1. On Days 5-7 and again on Days 9-11, half of the animals received morphine (Groups MS/SAL and MS/NLX) and half received saline (Groups SAL/SAL and SLA/NLX), as in Experiment 1. Testing, on Days 4, 8, and 12, was conducted as follows: Animals received a series of two injections at 10min intervals. Group MS/NLX $(n=8)$ received a morphine injection followed by a naloxone injection; Group MS/SAL $(n=8)$ received a morphine injection followed by a saline injection; Group SAL/NLX $(n=8)$ received a saline injection followed by a naloxone injection; Group SAL/SAL $(n=8)$ received two saline injections. Ten minutes later, the animals were placed in the running wheels for a 3-h session. The number of wheel revolutions was recorded every $20 \mathrm{~min}$.

\section{Results and Discussion}

Panels a, b, and c of Figure 2 show mean activity as a function of 20-min time blocks for all groups on Test Days 1, 2, and 3, respectively. Two effects are evident across test days. First, morphine-induced hyperactivity occurred on Test Days 2 and 3, but not on Test Day 1. This finding is consistent with previous results from our laboratory indicating that low doses of morphine elicit hyperactivity, which increases with exposure to morphine (Schnur, Bravo, \& Trujillo, 1983). Second, naloxone antagonism of morphine-induced hyperactivity increased across test days. This finding is congruent both with the dependence/withdrawal hypothesis and with the opponentprocess theory of motivation.

A $2 \times 2 \times 3 \times 9$ (first injection $\times$ second injection $\times$ test days $\times$ time blocks) mixed factorial ANOVA of locomotor activity indicated that the effects of test days $[F(2,56)=4.06, p<.025]$ and time blocks $[F(8,224)$ $=5.53, p<.001]$ were significant. In addition, the following interactions were significant: first injection $\times$ test days $[F(2,56)=8.29, p<.001]$, first injection $\times$ second injection $\times$ test days $[F(2,56)=5.55, p<.01]$, test days $\times$ time blocks $[F(16,448)=2.26, p<.01]$, first injection $\times$ test days $\times$ time blocks $[F(16,448)=1.69$, $p<.05$ ], and first injection $\times$ second injection $\times$ test days $\times$ time blocks $[F(16,448)=2.07, p<.01]$.

The effects of naloxone on morphine-elicited hyperactivity are evident in Panels b and c of Figure 2. A comparison of Groups MS/SAL and SAL/SAL on Test Days 2 and 3 shows morphine-elicited hyperactivity. This hyperactivity occurred earlier and reached a higher asymptote on Test Day 3 than on Test Day 2 (cf. Schnur, Bravo, \& Trujillo, 1983). Newman-Keuls tests $(p s<.05)$ indicated that, on Test Day 2, Group MS/SAL had a 

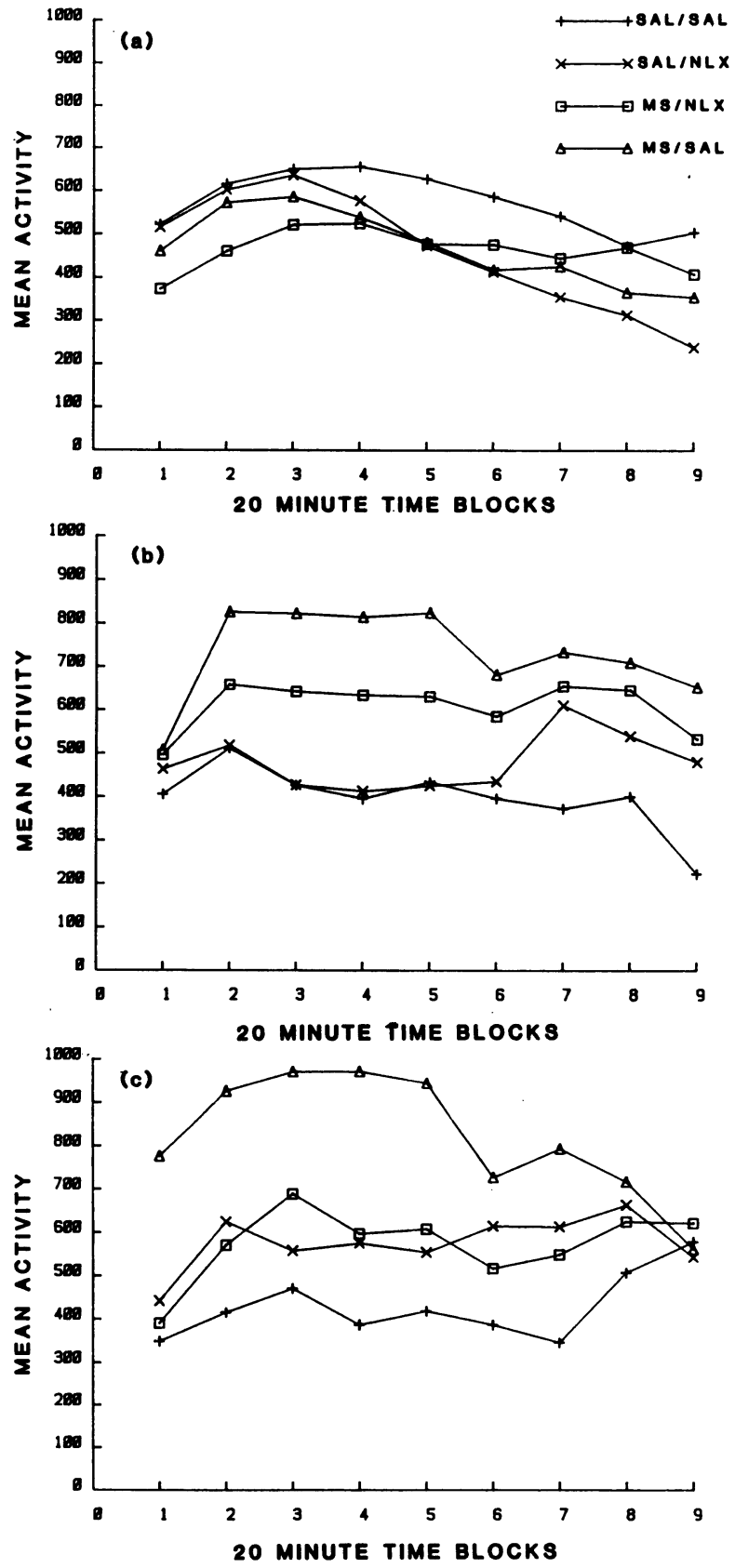

Figure 2. Mean running-wheel activity as a function of 20 -min time blocks for all groups in Experiment 2. Panels a, b, and c show results on Test Days 1, 2, and 3, respectively.

higher activity level than did Group SAL/SAL throughout the test session, except for the first $20 \mathrm{~min}$. Similarly, on Test Day 3, Group MS/SAL had a higher activity level than Group SAL/SAL throughout the test session, except for the final $\mathbf{4 0} \mathrm{min}$. Furthermore, naloxone antagonized morphine-elicited hyperactivity on both test days, but did not produce hypoactivity on either test day. On Test Day 2, Group MS/NLX was approximately $19 \%$ less active than Group MS/SAL during the first $2 \mathrm{~h}$ of the test session. On Test Day 3, Group MS/NLX was approximately $36 \%$ less active than Group MS/SAL during the first $2 \mathrm{~h}$ of the test session.

\section{GENERAL DISCUSSION}

The present results confirm and extend previous studies of naloxonemorphine interactions in the hamster. As previously reported, low doses of morphine elicit hyperactivity, whereas high doses elicit a biphasic time-effect pattern of hypoactivity followed by hyperactivity (Schnur, Bravo, Trujillo, \& Rocha, 1983). In Experiment 1, a 15-mg/kg dose of morphine led to a biphasic pattern on Test Days 1 and 3. In Experiment 2, a $2.5-\mathrm{mg} / \mathrm{kg}$ dose of morphine led to hyperactivity on Test Days 2 and 3 . In addition, the results of Experiment 1 confirm earlier findings (e.g., Schnur, 1985a) that naloxone $(0.4 \mathrm{mg} / \mathrm{kg})$ precipitates hypoactivity in morphine-treated animals (Group MS/NLX), but not in salinetreated controls (Group SAL/NLX).

The present results go beyond earlier studies by indicating that the effects of naloxone on morphine-elicited changes in hamster locomotor activity increase as a direct function of exposure to morphine. In Experiment 1 , naloxone-precipitated hypoactivity in morphine treated hamsters increased across 3 test days. In Experiment 2, naloxone antagonism of morphine-elicited hyperactivity also increased across 3 test days. These findings are consistent with the dependence/withdrawal hypothesis and with the opponent-process theory of motivation (Solomon \& Corbit, 1974).

\section{REFERENCES}

Brady, L. S., \& Holtzman, S. G. (1981). Locomotor activity in morphine-dependent and post-dependent rats. Pharmacology, Biochemistry \& Behavior, 14, 361-370.

Coussens, W. R., Crowder, W. F., \& Smith, S. G. (1973). Acute physical dependence upon morphine in rats. Behavioral Biology, 8 , 533-543.

Downs, D. A., \& Woods, J. H. (1976). Morphine, pentazocine and naloxone effects on responding under a multiple schedule of reinforcement in rhesus monkeys and pigeons. Journal of Pharmacology \& Experimental Therapeutics, 196, 298-306.

Goldberg, S. R., Morse, W. H., \& Goldberg, D. M. (1976). Some behavioral effects of morphine, naloxone and nalorphine in the squirrel monkey and the pigeon. Journal of Pharmacology \& Experimental Therapeutics, 196, 625-636.

JACOB, J. J., \& MiCHAUD, G. M. (1974). Acute physical dependence in the waking dog after a single low dose of norphine. Psychological Medicine, 4, 270-273.

Marcais, H., Bonnet, J. J., \& Costentin, J. (1981). Evidence for sedative effects of low doses of morphine in mice involving receptors insensitive to naloxone. Life Sciences, 28, 2737-2742.

SCHNUR, P. (1985a). Effects of naloxone and naltrexone on morphineelicited changes in hamster locomotor activity. Physiological Psychology, 13, 26-32.

SCHNUR, P. (1985b). Morphine tolerance and sensitization in the hamster. Pharmacology, Biochemistry \& Behavior, 22, 157-158.

SChNuR, P., Bravo, F., \& Trujlllo, M. (1983). Tolerance and sensitization to the biphasic effects of low doses of morphine in the hamster. Pharmacology, Biochemistry \& Behavior, 19, 435-439.

Schnur, P., Bravo, F., Trujllo, M., \& Rocha, S. (1983). Biphasic effects of morphine on locomotor activity in hamsters. Pharmacology, Biochemistry \& Behavior, 18, 357-361.

SchNUR, P., \& RAIGOZA, V. P. (1986a). Effects of naloxone on morphine induced sedation and hyperactivity in the hamster. Pharmacology, Biochemistry \& Behavior, 24, 849-854.

SchNuR, P., \& RaIGoza, V. P. (1986b). Evidence for an underlying opponent process during morphine elicited hyperactivity in the hamster. Life Sciences, 38, 1323-1329.

Solomon, R. L., \& CoRBit, J. D. (1974). An opponent-process theory of motivation: I. Temporal dynamics of affect. Psychological Review, 81, 119-145.

SteVens, D. R., \& KLEMm, W. R. (1979). Morphine-naloxone interactions: A role for nonspecific excitatory effects in withdrawal. Science, 205, 1379-1380.

Thornhill, J. A., Hirst, M., \& Gowdey, C. W. (1978). Changes in the hyperthermic responses of rats to daily injections of morphine and the antagonism of the acute response by naloxone. Canadian Journal of Physiology \& Pharmacology, 56, 483-489.

(Manuscript received for publication March 2, 1987.) 